\title{
Correlation and Path Coefficient Analysis for Some Yield-Related Traits in Pomegranate (Punica grantum L.) under Mid-Hill Conditions of Himachal Pradesh, India
}

\author{
Suparna Sinha ${ }^{1}$, Dinesh Singh Thakur ${ }^{2}$, Ankita Aman ${ }^{1}$, R.R. Singh ${ }^{1}$ and H. Mir ${ }^{1}$ \\ ${ }^{1}$ Department of Horticulture (Fruit and Fruit Tech.), Bihar Agricultural University, Sabour, \\ Bhagalpur-813210, India \\ ${ }^{2}$ Department of Horticulture, Dr. Y. S. P. U. H. \& F, Nauni, Solan-173230, India
}

*Corresponding author

\section{A B S T R A C T}

\section{Keywords \\ Factor analysis, Genetic diversity, Heritability, \\ Pomegranate \\ Article Info \\ Accepted: \\ 23 March 2018 \\ Available Online: \\ 10 April 2018}

Twenty pomegranates (Punica granatum L.) accessions were studied to determine inter-relationship of traits as well as to study genetic variation among yield attribution traits to understand the usefulness of genotypes in the breeding programme. It establishes that among all the parameters analyzed, a significant genetic difference was present for yield and yield attribution traits for exotic germplasm of pomegranate under study.

\section{Introduction}

Considering the increasing world population, expanding pomegranate cultivation may contribute towards providing the effective source of food and nutritional security.

Therefore, to sustain pomegranate industry in non-traditional areas one of the strategies is attention should be given on germplasm introduction. The pomegranate possesses a low genetic base and variability in India with a wide scope of being improved further, through a conventional or non-conventional method of breeding.

Characters of exotic germplasm of pomegranate are of great interest to breeder while those are complex in nature and also the result of the interaction of a number of components.

In the present study, an effort was represented to know the association and path analysis of component characters for fruit yield in pomegranate germplasms. 
Table.1 Correlation coefficients at genotypic $(\mathrm{G})$ and phenotypic $(\mathrm{P})$ levels with respect to fruit weight and yield component traits in twenty exotic pomegranate germplasms

\begin{tabular}{|c|c|c|c|c|c|c|c|c|c|c|c|c|c|c|c|c|c|c|c|c|}
\hline Character & & $\begin{array}{l}\text { Height } \\
\text { of Plant } \\
\text { (m) }\end{array}$ & $\begin{array}{c}\text { N-s } \\
\text { Sperad } \\
(\mathbf{m})\end{array}$ & E-w (m) & $\begin{array}{l}\text { Girth of } \\
\text { Stem } \\
(\mathbf{m m})\end{array}$ & $\begin{array}{l}\text { Numbers } \\
\text { of Stems }\end{array}$ & $\begin{array}{l}\text { Fruit } \\
\text { Length } \\
(\mathbf{m m})\end{array}$ & $\begin{array}{c}\text { Fruit } \\
\text { diameter } \\
(\mathbf{m m})\end{array}$ & $\begin{array}{l}\text { Yield/ } \\
\text { Plant } \\
\text { kg }\end{array}$ & $\begin{array}{c}\text { Rind } \\
\text { Thickness } \\
(\mathbf{m m})\end{array}$ & $\begin{array}{l}\text { Arils/ } \\
\text { Fruit }\end{array}$ & $\begin{array}{l}100 \text { Arils } \\
\text { Weight } \\
\text { (g) }\end{array}$ & $\begin{array}{l}\text { Aril/ } \\
\text { Rind } \\
\text { Ratio }\end{array}$ & $\begin{array}{c}\text { Aril } \\
\text { Length } \\
(\mathbf{m m})\end{array}$ & $\begin{array}{c}\text { Breadth } \\
(\mathrm{mm})\end{array}$ & $\begin{array}{l}\text { Juice } \\
\%\end{array}$ & $\begin{array}{l}\text { TSS } \\
(\%)\end{array}$ & $\begin{array}{c}\text { Tss/Acidity } \\
\text { Ratio }\end{array}$ & $\begin{array}{l}\text { Anthocyanins } \\
(\mathrm{mg} / 100 \mathrm{~g})\end{array}$ & $\begin{array}{l}\text { Phenols } \\
\text { (mg/100g) }\end{array}$ \\
\hline \multirow{2}{*}{ Height of Plant (m) } & G & 1.0000 & & & & & & & & & & & & & & & & & & \\
\hline & $\mathrm{P}$ & 1.0000 & & & & & & & & & & & & & & & & & & \\
\hline \multirow[t]{2}{*}{ N-s Sperad (m) } & G & 0.9055 & 1.0000 & & & & & & & & & & & & & & & & & \\
\hline & $\mathrm{P}$ & 0.6297 & 1.0000 & & & & & & & & & & & & & & & & & \\
\hline \multirow[t]{2}{*}{ E-w (m) } & G & 1.0129 & 0.9098 & 1.0000 & & & & & & & & & & & & & & & & \\
\hline & $\mathrm{P}$ & 0.6937 & 0.8839 & 1.0000 & & & & & & & & & & & & & & & & \\
\hline \multirow[t]{2}{*}{ Girth of Stem (mm) } & G & 0.7120 & 0.4471 & 0.4757 & 1.0000 & & & & & & & & & & & & & & & \\
\hline & $P$ & 0.4895 & 0.4429 & 0.4696 & 1.0000 & & & & & & & & & & & & & & & \\
\hline \multirow[t]{2}{*}{ Numbers of Stems } & G & -0.9227 & -0.9840 & -0.9443 & -0.9043 & 1.0000 & & & & & & & & & & & & & & \\
\hline & $P$ & 0.1874 & 0.2577 & 0.2787 & 0.1795 & 1.0000 & & & & & & & & & & & & & & \\
\hline \multirow[t]{2}{*}{ Fruit Length $(\mathbf{m m})$} & G & 0.2439 & 0.0830 & 0.2256 & 0.0630 & -0.8125 & 1.0000 & & & & & & & & & & & & & \\
\hline & $\mathrm{P}$ & 0.4514 & 0.0691 & 0.1124 & 0.0244 & -0.0544 & 1.0000 & & & & & & & & & & & & & \\
\hline \multirow{2}{*}{ Fruit diameter $(\mathrm{mm})$} & G & 0.2345 & -0.0688 & -0.0347 & -0.0509 & 0.2953 & 0.9822 & 1.0000 & & & & & & & & & & & & \\
\hline & $P$ & 0.1810 & -0.0677 & -0.0452 & -0.0493 & -0.0730 & 0.4793 & 1.0000 & & & & & & & & & & & & \\
\hline \multirow[t]{2}{*}{ Yield/ Plant kg } & G & 0.9940 & 0.7299 & 0.7960 & 0.4874 & -0.9632 & 0.6488 & 0.3159 & 1.0000 & & & & & & & & & & & \\
\hline & $P$ & 0.6952 & 0.7228 & 0.7848 & 0.4865 & 0.1468 & 0.3194 & 0.3083 & 1.0000 & & & & & & & & & & & \\
\hline \multirow[t]{2}{*}{ Rind Thickness (mm) } & G & 0.0869 & 0.0957 & 0.0355 & -0.1233 & -0.9694 & 0.5806 & 0.4587 & 0.1485 & 1.0000 & & & & & & & & & & \\
\hline & $\mathrm{P}$ & 0.0505 & 0.0876 & 0.0373 & -0.1229 & 0.1604 & 0.2935 & 0.4501 & 0.1475 & 1.0000 & & & & & & & & & & \\
\hline \multirow[t]{2}{*}{ Arils/ Fruit } & G & -0.0283 & -0.0372 & -0.0532 & 0.1478 & -0.1735 & 0.0781 & 0.3611 & -0.0932 & 0.2477 & 1.0000 & & & & & & & & & \\
\hline & $P$ & -0.0204 & -0.0353 & -0.0558 & 0.1475 & 0.0142 & 0.0406 & 0.3536 & -0.0925 & 0.2440 & 1.0000 & & & & & & & & & \\
\hline \multirow[t]{2}{*}{100 Arils Weight (g) } & G & 0.2192 & 0.0518 & 0.0907 & -0.2460 & 0.7779 & 0.0747 & -0.0730 & 0.3392 & -0.1639 & -0.6656 & 1.0000 & & & & & & & & \\
\hline & $P$ & 0.1542 & 0.0506 & 0.0888 & -0.2441 & -0.0669 & 0.0526 & -0.0667 & 0.3366 & -0.1538 & -0.6601 & 1.0000 & & & & & & & & \\
\hline \multirow[t]{2}{*}{ Aril/ Rind Ratio } & G & -0.0620 & -0.0134 & -0.1538 & 0.3274 & 0.9030 & -0.3986 & -0.2761 & 0.0507 & -0.3588 & 0.0730 & 0.0827 & 1.0000 & & & & & & & \\
\hline & $P$ & -0.0410 & -0.0125 & -0.1524 & 0.3263 & -0.1839 & -0.1866 & -0.2651 & 0.0506 & -0.3537 & 0.0723 & 0.0841 & 1.0000 & & & & & & & \\
\hline \multirow[t]{2}{*}{ Aril Length (mm) } & G & 0.3363 & 0.1874 & 0.2415 & -0.1544 & -0.1325 & 0.5106 & 0.5593 & 0.5969 & 0.3478 & 0.0182 & 0.3313 & -0.1370 & 1.0000 & & & & & & \\
\hline & $\mathrm{P}$ & 0.1284 & 0.1703 & 0.2218 & -0.1345 & -0.0419 & 0.1849 & 0.5212 & 0.5305 & 0.3172 & 0.0123 & 0.2962 & -0.1275 & 1.0000 & & & & & & \\
\hline \multirow[t]{2}{*}{ Breadth $(\mathrm{mm})$} & G & 0.9016 & 0.4491 & 0.4964 & 0.1499 & -0.7308 & 0.9333 & 0.7409 & 0.9188 & 0.4160 & -0.1092 & 0.4218 & -0.0912 & 1.0849 & 1.0000 & & & & & \\
\hline & $P$ & 0.0510 & 0.2614 & 0.2966 & 0.0963 & -0.0465 & 0.0123 & 0.4553 & 0.5560 & 0.2291 & -0.0685 & 0.2484 & -0.0579 & 0.6915 & 1.0000 & & & & & \\
\hline \multirow[t]{2}{*}{ Juice \% } & G & 0.4870 & 0.2177 & 0.2710 & 0.0600 & 0.9272 & 0.7003 & 0.2433 & 0.4517 & -0.1539 & -0.2397 & 0.2270 & -0.0159 & 0.2472 & 0.4565 & 1.0000 & & & & \\
\hline & $P$ & 0.3430 & 0.2159 & 0.2684 & 0.0593 & -0.1397 & 0.3471 & 0.2347 & 0.4511 & -0.1493 & -0.2389 & 0.2269 & -0.0147 & 0.2140 & 0.2634 & 1.0000 & & & & \\
\hline \multirow[t]{2}{*}{ TSS (\%) } & G & 0.3759 & 0.2417 & 0.1049 & 0.2833 & -0.8937 & 0.6139 & 0.4418 & 0.3014 & 0.1534 & 0.1553 & -0.1846 & -0.0658 & 0.3256 & 0.4417 & 0.2799 & 1.0000 & & & \\
\hline & $P$ & 0.1702 & 0.2311 & 0.0916 & 0.2689 & 0.0013 & 0.2247 & 0.3960 & 0.2856 & 0.1329 & 0.1500 & -0.1782 & -0.0660 & 0.2719 & 0.3225 & 0.2622 & 1.0000 & & & \\
\hline \multirow[t]{2}{*}{ Tss/Acidity Ratio } & G & 0.5652 & -0.0180 & 0.0892 & 0.2724 & 0.6723 & 0.8415 & 0.3420 & 0.4803 & -0.1674 & -0.1936 & 0.4404 & 0.2401 & 0.3828 & 0.5975 & 0.5437 & 0.4904 & 1.0000 & & \\
\hline & $P$ & 0.1523 & -0.0145 & 0.0575 & 0.2291 & -0.1091 & 0.1566 & 0.2651 & 0.3880 & -0.1511 & -0.1605 & 0.3467 & 0.1851 & 0.3748 & 0.5620 & 0.4413 & 0.3948 & 1.0000 & & \\
\hline \multirow[t]{2}{*}{ Anthocyanins (mg/100g) } & G & 0.0293 & 0.2662 & 0.2733 & -0.0143 & 0.4974 & 0.0327 & 0.0530 & 0.1522 & 0.0135 & -0.1315 & -0.1659 & -0.1209 & 0.0686 & 0.2358 & 0.0635 & -0.3428 & -0.2314 & 1.0000 & \\
\hline & $P$ & 0.0488 & 0.2655 & 0.2704 & -0.0151 & -0.0514 & 0.0579 & 0.0504 & 0.1525 & 0.0140 & -0.1307 & -0.1640 & -0.1200 & 0.0500 & 0.1011 & 0.0645 & -0.3305 & -0.2127 & 1.0000 & \\
\hline Phenols (mg/100g) & G & -0.1620 & 0.1479 & 0.0517 & 0.0197 & 0.0286 & -0.1211 & -0.2086 & -0.0929 & 0.2331 & 0.2752 & -0.3605 & 0.2298 & -0.1184 & -0.3376 & -0.3991 & 0.0419 & -0.5074 & 0.0005 & 1.0000 \\
\hline & $P$ & -0.1133 & 0.1483 & 0.0489 & 0.0199 & 0.0088 & -0.0596 & -0.2031 & -0.0924 & 0.2295 & 0.2753 & -0.3569 & 0.2293 & -0.1044 & -0.2021 & -0.3975 & 0.0375 & -0.4136 & 0.0005 & 1.0000 \\
\hline Fruit Weight (g) & G & 0.4386 & 0.0717 & 0.1754 & -0.0342 & 0.9886 & 0.5955 & 0.5879 & 0.3864 & 0.0963 & 0.3625 & 0.2741 & -0.0690 & 0.3659 & 0.4748 & 0.1823 & 0.0260 & 0.2610 & -0.1099 & -0.1429 \\
\hline & $\mathrm{P}$ & 0.3415 & 0.0749 & 0.1751 & -0.0341 & -0.1136 & 0.3333 & 0.5748 & 0.3831 & 0.0925 & 0.3584 & 0.2697 & -0.0673 & 0.3251 & 0.2547 & 0.1809 & 0.0121 & 0.1956 & -0.1022 & -0.1407 \\
\hline
\end{tabular}

*For value $>0.378 \& * *$ for value $>0.586$ 
Table.2 Estimates of direct and indirect effects of different twenty traits on fruit weight of pomegranate germplasm

\begin{tabular}{|c|c|c|c|c|c|c|c|c|c|c|c|c|c|c|c|c|c|c|c|c|c|c|}
\hline No & Character & $\begin{array}{l}\text { Height of } \\
\text { Plant (m) }\end{array}$ & $\underset{(\mathrm{m})}{\mathrm{N}-\mathrm{s} \text { Sperad }}$ & E-w(m) & $\begin{array}{c}\text { Girth of } \\
\text { Stem }(\mathbf{m m})\end{array}$ & $\begin{array}{c}\text { Number of } \\
\text { Stems }\end{array}$ & $\begin{array}{c}\text { f } \begin{array}{c}\text { Fruit } \\
\text { Length } \\
\text { (mm) }\end{array}\end{array}$ & $\begin{array}{c}\text { Fruit } \\
\text { diameter } \\
(\mathbf{m m})\end{array}$ & $\begin{array}{c}\text { Yield/ Plant } \\
\text { kg }\end{array}$ & $\begin{array}{c}\text { Rind } \\
\text { Thickness } \\
(\mathbf{m m})\end{array}$ & Arils/ Fruit & $\begin{array}{c}100 \\
\text { Arils } \\
\text { Weight (g) }\end{array}$ & $\begin{array}{c}\text { Aril/ Rind } \\
\text { Ratio }\end{array}$ & $\begin{array}{l}\text { Aril Length } \\
(\mathbf{m m})\end{array}$ & $\begin{array}{c}\text { Breadth } \\
(\mathbf{m m})\end{array}$ & Juice\% & TSS(\%) & $\begin{array}{l}\text { Tss/Acidity } \\
\text { Ratio }\end{array}$ & $\begin{array}{c}\text { Antho } \\
\text { cyanins } \\
\text { (mg/100g) }\end{array}$ & $\begin{array}{c}\text { Phenols } \\
\text { (mg/100g) }\end{array}$ & $\mathbf{r}_{\mathrm{Xy}}$ & \\
\hline & & X1 & $\mathrm{X} 2$ & X3 & X4 & X5 & X6 & $\mathrm{X} 7$ & X8 & X9 & X10 & X11 & X12 & X13 & X14 & X15 & X16 & X17 & X18 & X19 & & \\
\hline X1 & $\begin{array}{l}\text { Height of } \\
\text { Plant(m) }\end{array}$ & -0.3313 & -0.1664 & 0.8349 & 0.4641 & 0.0460 & -0.0237 & 0.2180 & -0.8046 & -0.0037 & -0.0289 & 0.3626 & 0.0091 & 0.0506 & -0.3606 & 0.2276 & 0.0001 & -0.0113 & 0.0074 & -0.0511 & 0.4386 & \\
\hline $\mathrm{X} 2$ & $\begin{array}{l}\text { N-sSperad } \\
\text { (m) }\end{array}$ & -0.3000 & -0.1838 & 0.7499 & 0.2914 & 0.0475 & -0.0081 & -0.0640 & -0.5908 & -0.0041 & -0.0380 & 0.0857 & 0.0020 & 0.0282 & -0.1603 & 0.1017 & 0.0000 & 0.0004 & 0.0673 & 0.0467 & 0.0717 & \\
\hline $\mathbf{X} 3$ & E-w(m) & -0.3356 & -0.1672 & 0.8242 & 0.3101 & 0.0561 & -0.0219 & -0.0322 & -0.6443 & -0.0015 & -0.0544 & 0.1501 & 0.0227 & 0.0363 & -0.1772 & 0.1266 & 0.0000 & -0.0018 & 0.0691 & 0.0163 & 0.1754 & \\
\hline $\mathrm{X} 4$ & $\begin{array}{l}\text { Girth of } \\
\text { Stem(mm) }\end{array}$ & -0.2359 & -0.0822 & 0.3921 & 0.6518 & 0.0384 & -0.0061 & -0.0473 & -0.3945 & 0.0053 & 0.1510 & -0.4070 & -0.0482 & -0.0232 & -0.0535 & 0.0280 & 0.0000 & -0.0054 & -0.0036 & 0.0062 & -0.0342 & \\
\hline X5 & $\begin{array}{l}\text { Numbers of } \\
\text { Stems }\end{array}$ & 0.6370 & 0.3647 & -1.9322 & -1.0457 & -0.0239 & 0.0790 & 0.2745 & 1.0225 & 0.0717 & -0.1773 & 1.2870 & -0.2508 & -0.0199 & 0.2608 & 0.6201 & -0.0001 & -0.0134 & 0.1257 & 0.0090 & 0.9886 & \\
\hline X6 & $\begin{array}{l}\text { Fruit Length } \\
(\mathrm{mm})\end{array}$ & -0.0808 & -0.0153 & 0.1859 & 0.0411 & 0.0195 & -0.0972 & 0.9130 & -0.5252 & -0.0249 & 0.0798 & 0.1236 & 0.0587 & 0.0768 & -0.4401 & 0.3272 & 0.0001 & -0.0168 & 0.0083 & -0.0382 & 0.5955 & \\
\hline $\mathrm{X} 7$ & $\begin{array}{ll}\begin{array}{l}\text { Fruit } \\
(\mathrm{mm})\end{array} & \text { Diameter }\end{array}$ & -0.0777 & 0.0127 & -0.0286 & -0.0332 & -0.0071 & -0.0955 & 0.9296 & -0.2557 & -0.0197 & 0.3689 & -0.1208 & 0.0407 & 0.0841 & -0.2644 & 0.1137 & 0.0001 & -0.0068 & 0.0134 & -0.0658 & 0.5879 & \\
\hline $\mathrm{X8}$ & Yield/Plant kg & -0.3294 & -0.1342 & 0.6561 & 0.3177 & 0.0303 & -0.0631 & 0.2937 & -0.8094 & -0.0064 & -0.0952 & 0.5611 & -0.0075 & 0.0898 & -0.3279 & 0.2111 & 0.0001 & -0.0096 & 0.0385 & -0.0293 & 0.3864 & \\
\hline X9 & $\begin{array}{l}\text { Rind Thickness } \\
(\mathrm{mm})\end{array}$ & -0.0288 & -0.0176 & 0.0293 & -0.0804 & 0.0400 & -0.0564 & 0.4264 & -0.1202 & -0.0429 & 0.2531 & -0.2712 & 0.0529 & 0.0523 & -0.1485 & -0.0719 & 0.0000 & 0.0033 & 0.0034 & 0.0735 & 0.0963 & \\
\hline X10 & Arils/Fruit & 0.0094 & 0.0068 & -0.0439 & 0.0963 & 0.0042 & -0.0076 & 0.3357 & 0.0754 & -0.0106 & 1.0216 & -1.1012 & -0.0107 & 0.0027 & 0.0390 & -0.1120 & 0.0000 & 0.0039 & -0.0332 & 0.0868 & 0.3625 & \\
\hline X11 & $\begin{array}{l}\text { 100Arils } \\
\text { Weight(g) }\end{array}$ & -0.0726 & -0.0095 & 0.0748 & -0.1604 & -0.0186 & -0.0073 & -0.0679 & -0.2745 & 0.0070 & -0.6800 & 1.6544 & -0.0122 & 0.0498 & -0.1505 & 0.1061 & 0.0000 & -0.0088 & -0.0419 & -0.1138 & 0.2741 & \\
\hline X12 & $\begin{array}{l}\text { Aril/Rind } \\
\text { Ratio }\end{array}$ & 0.0205 & 0.0025 & -0.1268 & 0.2134 & -0.0408 & 0.0388 & -0.2567 & -0.0411 & 0.0154 & 0.0745 & 0.1369 & -0.1473 & -0.0206 & 0.0326 & -0.0074 & 0.0000 & -0.0048 & -0.0306 & 0.0725 & -0.069 & \\
\hline $\mathrm{x} 13$ & $\begin{array}{l}\text { Aril Length } \\
(\mathrm{mm})\end{array}$ & -0.1114 & -0.0345 & 0.1990 & -0.1007 & 0.0032 & -0.0496 & 0.5199 & -0.4832 & -0.0149 & 0.0186 & 0.5481 & 0.0202 & 0.1504 & -0.3872 & 0.1155 & 0.0001 & -0.0076 & 0.0174 & -0.0374 & 0.3659 & \\
\hline X14 & $\begin{array}{l}\text { Breadth } \\
(\mathrm{mm})\end{array}$ & -0.3349 & -0.0826 & 0.4092 & 0.0977 & 0.0175 & -0.1199 & 0.6888 & -0.7437 & -0.0179 & -0.1116 & 0.6978 & 0.0134 & 0.1632 & -0.3569 & 0.2133 & 0.0001 & -0.0119 & 0.0596 & -0.1065 & 0.4748 & \\
\hline X15 & Juice\% & -0.1614 & -0.0400 & 0.2234 & 0.0391 & -0.0318 & -0.0681 & 0.2262 & -0.3656 & 0.0066 & -0.2449 & 0.3756 & 0.0023 & 0.0372 & -0.1629 & 0.4673 & 0.0000 & -0.0108 & 0.0161 & -0.1259 & 0.1823 & \\
\hline X16 & $\operatorname{TSS}(\%)$ & -0.1245 & -0.0444 & 0.0865 & 0.1846 & 0.0214 & -0.0597 & 0.4107 & -0.2440 & -0.0066 & 0.1587 & -0.3054 & 0.0097 & 0.0490 & -0.1576 & 0.1308 & 0.0002 & -0.0098 & -0.0867 & 0.0132 & 0.0260 & \\
\hline X17 & $\begin{array}{l}\text { Tss/Acidity } \\
\text { Ratio }\end{array}$ & -0.1873 & 0.0033 & 0.0736 & 0.1776 & -0.0161 & -0.0818 & 0.3179 & -0.3888 & 0.0072 & -0.1978 & 0.7286 & -0.0354 & 0.0576 & -0.2132 & 0.2541 & 0.0001 & -0.0199 & -0.0585 & -0.1601 & 0.2610 & \\
\hline X18 & $\begin{array}{l}\begin{array}{l}\text { Anthocyani } \\
(\mathrm{mg} / 100 \mathrm{~g})\end{array} \\
\mathrm{ns}\end{array}$ & $\leqslant-0.0097$ & -0.0489 & 0.2253 & -0.0093 & -0.0119 & -0.0032 & 0.0493 & -0.1232 & -0.0006 & -0.1343 & -0.2745 & 0.0178 & 0.0103 & -0.0842 & 0.0297 & -0.0001 & 0.0046 & 0.2528 & 0.0002 & -0.1099 & \\
\hline \multirow[t]{3}{*}{ X19 } & $\begin{array}{l}\text { Phenols } \\
\text { (mg/100g) }\end{array}$ & 0.0537 & -0.0272 & 0.0426 & 0.0129 & -0.0007 & 0.0118 & -0.1939 & 0.0752 & -0.0100 & 0.2811 & -0.5965 & -0.0339 & -0.0178 & 0.1205 & -0.1865 & 0.0000 & 0.0101 & 0.0001 & 0.3155 & -0.1429 & \\
\hline & & & & & & & & & & & & & & & & & & & & & $\mathrm{R}^{2}$ & 0.6931 \\
\hline & & & & & & & & & & & & & & & & & & & & & R.E. & 0.7208 \\
\hline
\end{tabular}




\section{Materials and Methods}

Twenty pomegranate germplasm having diversity for various morphological characters (during flowering, growth condition of the plant, fruits, and quality) were taken in a randomized block design with three replication at Experimental farm of Fruit Science, Dr. Y. S. Parmar University of Horticulture and Forestry, Nauni, Solan (Himachal Pradesh, India) during 2013. Plant protection means were also applied uniformly for all the plots during the period of the experiment. Five representative fruits were taken in each replicates at optimum maturity. The phenotypic and genotypic correlation coefficients were estimated as per Burton (1952). Direct and indirect paths analyses at a genotypic level were obtained by according to Dewey and Lu (1959).

\section{Results and Discussion}

Commonly, the value of genotypic correlations is greater than phenotypic correlations (Table 1). This indicates that there are inherent associations among various traits and environment had not played much sole in reducing their actual association. Fruit weight showed positive and significant correlations number of stems at genotypic level. Ece and Darakci (2007) in tomato reported positive correlations of fruit weight with number of stem. Also, Fruit diameter had positive and highly significant correlation with fruit weight at phenotypic and genotypic levels. Positive association of fruit weight with fruit diameter have been reported by Pandey and Bist (1998). Hence, selection of germplasm(s) with number of stems per plant, and fruit diameter would be effective to obtain higher fruit weight.

Path coefficient analysis provides a realistic ground for allocation of appropriate weightage to different independent traits while designing a pragmatic plan for improving fruit weight. The data on path coefficient analysis at genotypic level showed the direct and indirect effects of important traits over fruit weight had been presented in Table 2. The data revealed that fruit weight has a maximum positive direct effect on 100 arils weight (1.6544) followed by arils/fruit (1.0216), fruit breadth (0.9296), E-W spread (0.8242), girth of stems $(0.6518)$, juice \% $(0.4673)$ and phenols (0.3155). Maximum negative effect of yield (-0.8094), aril breadth $(-0.3569)$ and height of plant $(-0.3313)$ was observed on fruit weight. The residual effect value $(0.7208)$ shows that there are few other possible independent traits which were not included in this study. By path coefficient analysis observation, it can be concluded that these traits could be utilized by breeders as selection criteria to select the accessions influencing fruit weight. The direct effect of yield is negative for fruit weight, but the correlation coefficient is positive which shows we can get best results by applying selection indices.

Path analysis revealed that 100 arils weight followed by arils/fruit, fruit breadth, E-W spread, girth of stem, juice \%, and phenols are the strongest forces influencing fruit weight.

\section{References}

Ali Ece and Nilay Darakci, 2007. Determination of relationships between number of stem and yield of tomato (Lycopersicon lycopersicum L.). Asian Journal of Plant Sciences, 6: 802-808.

Burton, G.W. 1952. Quantitative inheritance in grasses. In: Proc. $6^{\text {th }}$ Int. Grassland Cong. 1: 277-83.

Burton, G.W. and De Vane, E.H. 1953. Estimating heritability in tall fescue (Festuca arundinacea) from replicated clonal material. Agron. J. 45: 478-81. 
Dewey, D.R., and Lu. K.H. 1959. A correlation and path coefficient analysis of components of crested wheatgrass seed production. Agron. J. 51: 511-18.

Pandey G and Bist H S (1998). Variability, correlation and path analysis in pomegranate germplasm. Hort. J. 11: 712

\section{How to cite this article:}

Suparna Sinha, Dinesh Singh Thakur, Ankita Aman, R.R. Singh and Mir, H. 2018. Correlation and Path Coefficient Analysis for Some Yield-Related Traits in Pomegranate (Punica grantum L.) under Mid-Hill Conditions of Himachal Pradesh, India. Int.J.Curr.Microbiol.App.Sci. 7(04): 2816-2820. doi: https://doi.org/10.20546/ijcmas.2018.704.320 